\title{
Urgences
}

\section{Liminaire (à deux voix, bien sûr)}

\section{Robert Dion et Renald Bérubé}

Numéro 25, octobre 1989

Multiples de Hamlet

URI : https://id.erudit.org/iderudit/025539ar

DOI : https://doi.org/10.7202/025539ar

Aller au sommaire du numéro

Éditeur(s)

Urgences

ISSN

0226-9554 (imprimé)

1927-3924 (numérique)

Découvrir la revue

Citer ce document

Dion, R. \& Bérubé, R. (1989). Liminaire (à deux voix, bien sûr). Urgences, (25),

5-11. https://doi.org/10.7202/025539ar d'utilisation que vous pouvez consulter en ligne.

https://apropos.erudit.org/fr/usagers/politique-dutilisation/ 


\section{Liminaire (à deux voix, bien sûr)}

\section{Hypertextualité: conversions et conversations}

1) L'étude des relations entre l'hypertexte et l'hypotexte introduit au problème fondamental de la coexistence des énoncés au sein du champ littéraire. Le texte de départ (l'hypotexte) et le texte d'arrivée (l'hypertexte) ne sont pas liés que par des rapports de contiguïté, c'est-à-dire des rapports de parenté formelle et thématique plus ou moins étroits. Dans le cas qui nous occupe, nous avons plutôt affaire à une synthèse bitextuelle, ce qui revient à dire que l'hypertexte est le résultat d'un syncrétisme. En effet, le texte d'arrivée évoque et transforme le texte de départ. Bien que totalement incorporé à l'hypertexte, l'hypotexte conserve néanmoins quelque chose de sa forme originelle, ce qui lui permet d'être reconnu comme tel.

Même s'il demeure reconnaissable, l'hypotexte n'en est pas moins profondément modifié par le processus intertextuel, que ce dernier soit ou non parodique. L'hypotexte ne saurait se tirer indemme de la conversion et de la conversation des textes. Comme l'écrivait Anthony Wall dans un article récent ${ }^{1}$, l'hypotexte reste marqué par les assauts de l'hypertexte. Il ne retrouve jamais sa pureté originelle. Aussi peut-on affirmer (plaisamment) qu'il n'est guère possible de lire le Hamlet de Shakespeare sans tenir compte de celui de Laforgue, non plus que l'inverse. Si le second ne se conçoit pas sans le premier, celui-ci ne peut pas davantage être lu sans être considéré, peu ou prou, comme le modèle de celui-là.

S'ouvre, ici, un espace vertigineux, qui est celui de la circulation des textes.

2) L'hypotexte et l'hypertexte sont marqués de façon indélébile par leur coexistence. Linterprétation du premier est à jamais conditionnée par l'existence du second. On ne peut pas imaginer de réécriture hypertextuelle qui soit neutre: c'est pourquoi la parodie est une forme privilégiée d'hypertextualité.

1 Anthony Wall, "Vers une notion de la colle parodique", Études fitteraires, Québec, vol. XIX, n 1 (printemps-été 1986), p. 21-36. Je me permets de renvoyer à Robert Dion et Marty Laforest, “ Avatars de la parodie: définitions et applications du concept de "parodie" ", RSSI, Toronto, vol. VII, $n^{\circ} 1$ (1987), p. 73-87. 
6

L'insertion de l'hypotexte dans le texte d'arrivée, qu'il s'agisse de citations ou de plagiats, suffit à en modifier la lecture, peu importe que l'intention soit ou non parodique. Daniel Bilous note judicieusement que la parodie la plus efficace serait celle qui, à la limite, se contenterait « de transposer en contexte ingrat le mot d'autrui, de le dépayser mais en le citant littéralement ${ }^{2}$. Cette intuition rappelle celle de Borges dans son Pierre Ménard. Elle pose la question du statut des textes: doit-on postuler une hiérarchie des textes mis en circulation dans le processus intertextuel? le premier texte - le texte le plus ancien, c'est-à-dire l'hypotexte - estil nécessairement le texte premier? qu'en est-il des cas, au demeurant nombreux, où l'hypertexte, généralement considérẻ comme le texte parasitaire, s'avère le plus réussi? Ces questions sont capitales. Elles remettent en cause une conception de l'intertextualité et plus particulièrement de la parodie qui tend à valoriser l'hypotexte au détriment de l'hypertexte, assimilé à une protubérance disgracieuse, voire à un monstre sémiotique.

3) Au vu de ce qui précède, il y a lieu de se défier de la métaphore du palimpseste, bien que celle-ci s'avère fort séduisante. Comme toutes les métaphores, elle est prise dans un mouvement contradictoire: elle fait surgir des éclairs fulgurants et en même temps elle fait obstacle à une connaissance véritable des faits observés.

Dans le palimpseste, le texte nouveau cache le texte ancien, de sorte que leur interaction est réduite au minimum. La primauté du texte second est absolue. Or, nous avons vu que le phénomène de l'hypertextualité modifie profondément les deux textes en cause. Les traces de la rencontre persistent dans les deux textes, un peu à la manière des phosphènes. Tout texte engagé dans un rapport hypertextuel ne peut légitimement être lu sans le concours de l'autre texte. Un palimpseste, en revanche, est d'autant plus lisible que les traces du texte ancien sont moins apparentes. Sur le vélin, sont gommées les traces du texte premier, que le texte second vient, à proprement parler, remplacer.

Somme toute, la métaphore du palimpseste ne désigne qu'une frange d'un phénomène beaucoup plus large qui reste

2 Daniel Bilous, "Intertexte/pastiche: l'intermimotexte ", Texte, Toronto, $\mathrm{n}^{\circ} 2$ (1983), p. 138. 
partiellement à décrire. Nous souhaitons bien évidemment que ce numéro d'Urgences contribue à faire avancer nos connaissances.

\section{Robert Dion}

\section{Hamlet et sa descendance}

Un éminent professeur d'Oxford le disait déjà il y a bien des années: "quiconque se mettrait en tête de lire tout 'ce qu'on a écrit sur Hamlet n'aurait plus le temps de rien lire d'autre, pas même Hamlet".

Ce qu'à Dieu ne plaise!

Michel Grivelet 1

"Ce qu'à Dieu ne plaise!", en effet. Sinon, s'agissant de la masse critique ou de la critique massive dont Hamlet a été et demeure l'objet - ce numéro d'Urgences ajoutant à ladite masse -, le risque serait grand d'une sorte d'enterrement de classe première et au demeurant fort brillante pour l'œuvre dont le Prince danois est le héros. Le texte critique, hypertrophique, risquerait alors, impérialiste, d'occulter la voix dont il entend parler, la voix depuis laquelle parle sa sienne; d'autant plus que la critique de la critique (ou le métatexte du métatexte) a aussi ses droits, ce qui ne saurait en rien nuire au danger d'hypertrophie auquel il vient d'être fait allusion.

Et puis non; peut-être vaut-t-il mieux oublier les lignes qui précèdent, le danger n'étant pas aussi grand qu'on pourrait le croire en dépit de la susdite masse évoquée: le Hamlet shakespearien, même si le temps, critique ou autre, peut sembler hors de ses gonds, tient bien en mains les rênes de son royaume dramaturgique. À preuve qu'on ne saurait l'enterrer et quelle que soit la classe des fossoyeurs, dirait un ami forcément paradoxal du Prince, la masse critique ellemême: on n'en finit pas, malgré la multiplicité des textes

1 Michel Grivelet, Marie-Madeleine Martinet et Dominique Goy-Blanquet, Shakespeare de A a Z... ou presque, Paris, Aubier, 1988, p. 9. 
critiques qui démontrent précisément cela, de revenir à la pièce de Shakespeare. Si l'"hamletologie", selon le mot d'André Green ${ }^{2}$, semble bien devenue une "discipline", Hamlet en demeure le seigneur souverain; là où Hamlet s'interroge: "Être ou ne pas être", Hamlet est. Et sa voix, mourante à la fin de l'œuvre, n'en finit pas de répondre à ceux et à celles qui l'interrogent.

S’il y a les textes critiques, praticiens du métatexte, il y a aussi, nés depuis Hamlet, les textes de création, praticiens de l'hypertexte ou de l'hypertextualité. C'est-à-dire que Hamlet n'a pas donné naissance qu'à des études ou des analyses; il se trouve aussi à l'origine de textes de création - romans, poèmes, nouvelles ou œuvres théâtrales de plein droit. Dont il serait bien difficile de dire si l'"éminent professeur d'Oxford" cité par Grivelet les compte parmi «tout ce qu'on a écrit sur Hamlet ". Or qui écrit d'après écrit aussi sur: l'hypertexte relève toujours aussi du métatexte, le Genette de Palimpsestes l'a assez souligné. Et Robert Dion, dans la première partie de ce liminaire, a bien montré que la coexistence hypotexte-hypertexte, toute pacifique qu'elle puisse être, a des effets et sur l'un et sur l'autre -texte. L'hyper- ne saurait être orphelin de son ou de ses ancêtres, l'hypo- ne saurait demeurer innocent de sa descendance.

Or, la descendance hypertextuelle de Hamlet est à la fois nombreuse et variée, rusée ou manifeste. Nous n'en donnerons ici, à titre indicatif et en vrac, que quelques exemples:

Hamlet à Wittenberg, de Gerhart Hauptmann;

Hamlet ou la longue nuit prend fin, d'Alfred Döblin;

Une nuit avec Hamlet, de Vladimir Holan;

Hamlet des faubourgs, de Bernard Kops;

Hamlet-Machine, de Heiner Müller;

Rosencrantz et Guildenstern sont morts, de Tom Stoppard;

Hamlet de Tarascon, de Jean Canolle;

Hamlet et les sept nains, de Patrick Roegier;

«Hamlet» (dans Moralités légendaires), de Jules Laforgue;

2 André Green, Hamlet et Hamlet. Une interprétation psychanalytique de la représentation, Paris, Balland, 1982, p. 13. 
Mouchoir de nuages, de Tristan Tzara;

La machine infernale, de Jean Cocteau;

L'embrumé, de Viard et Zacharias;

Hamlet, de Gotlib.

Et il n'est fait mention ni du Lorenzaccio de Musset ni du Erik XIV de Strindberg; ni de l'"Ophélie" (dans Poésies) de Rimbaud ni de "L'accent grave" (dans Paroles) de Jacques Prévert; ni de la présence de Hamlet dans l'Ulysse de Joyce ou dans Un rude hiver de Raymond Queneau. Hamlet est pourtant là, aux frontières mouvantes de l'hypertextualité et des autres manifestations de la transtextualité - comme dans Mouchoir de nuages et dans La machine infernale, oeuvres mentionnées.

La traduction étant bien aussi, selon Genette, une pratique hypertextuelle, il faudrait encore mentionner toutes les traductions/adaptations de l'oeuvre de Shakespeare qui, depuis Jean-François Ducis jusqu'à Yves Bonnefoy en passant par Alexandre Dumas, André Gide ou Vercors - pour rester dans le seul domaine français - ont présenté leur version de la tragédie se déroulant à Elseneur. De même, il faudrait aussi prendre en considération, par exemple, les "textes crayonnés" par Mallarmé et les traces laissées dans son oeuvre poétique par le héros danois. Pour qui l'exercice de la pensée, de ses nuances et de ses paradoxes, est une activité fondamentale; pour qui l'exercice de l'écriture est le lieu d'une réflexion qui se traduit en art poétique ou dramatique, Hamlet, avec ses jeux de miroirs, ses conseils aux comédiens et sa mise en abyme canonique, demeure le lieu par excellence d'un travail toujours inachevé, plein de surprises et de découvertes. Le lieu premier du jeu des questions et des réponses, le lieu éternel du jeu des mêmes.

S'agissant de Hamlet, la littérature québécoise, issue d'un pays qui sait ce qu' «être ou ne pas être " veut dire, d'un pays dont les habitants sont des «agents doubles" disait Hubert Aquin, la littérature québécoise, donc, n'est certes pas en reste. À preuve, le trio hamlétien suivan t: Hamlet, d'après Thomas Kyd (!), téléthéâtre de Jacques Languirand diffusé par Radio-Canada en 1956; Hamlet, Prince du Québec, pièce de Robert Gurik créée en 1968; Neige noire de Hubert Aquin, roman publié en 1974. Un téléthéâtre d'apprentissage, comme on dit un roman du même "genre", écrit dans une tonalité 
qui peut le rapprocher de l'Antigone d'Anouilh; écrit, surtout, d'après une pièce perdue depuis longtemps! Puis une pièce éminemment politique, écrite dans le sillage du «Vive le Québec libre!" prononcé par le général De Gaulle depuis le balcon de l'Hôtel de Ville de Montréal en 1967. Enfin, un roman (policier?) qui est aussi un scénario (autobiographique, du point de vue de Nicolas?); roman/scénario au cours duquel nous assistons à une répétition et à une représentation du Hamlet de Shakespeare, ledit roman/scénario (qui sait pratiquer le centon) étant par ailleurs enchâssé dans un commentaire plutôt métatextuel qui est une longue réflexion sur le temps et sur les rapports entre l'écriture romanesque et l'écriture cinématographique. Oh Hamlet, que de textes on commet en ton nom!

$* * *$

Le numéro d'Urgences qu'on va lire, la lecture de ce liminaire duel/dialogique s'achevant, peut se présenter ainsi: enchâssées entre deux textes qui analysent les limites premières ('inconscient, avec les réserves qu'apporte Jean Bellemin-Noël) et dernières (le plagiat, ainsi qu'en parle Marilyn Randall) de la pratique hypertextuelle ou intertextuelle, quatre études portant sur des oeuvres dans lesquelles Hamlet joue un rôle de premier plan. L'inconscient pourrait-il être une sorte d'hypotexte... informulé, se demande BelleminNoël; dans le cadre de ce numéro, il est bon de rappeler que l'inconscient évoque presque irrésistiblement l'œdipe freudien et que celui-ci, en ses propres origines de 1897, doit presque autant à Hamlet qu'à CEdipe roi. Et que La machine infernale, lue ou vue sous cet angle, est une oeuvre particulièrement brillante et réussie. Et le plagiat, s'agissant de la réplique refondue d'une oeuvre? Marilyn Randall montre bien que, sur ce plan, tout se joue dans l'affichage ou la dissimulation du pacte ou du contrat. Quel pacte ou contrat, se demande sans doute la descendance héritière de Margaret Mitchell et, donc, des droits d'Autant en emporte le vent, peut bien lier le célèbre roman sudiste et La bicyclette bleue de Régine Deforges? La justice (littéraire?) devra trancher.

Les études enchâssées: du "Hamlet" de Jules Laforgue, par Sophie Lemieux; de Mouchoir de nuages de Tristan 
Tzara, par Martine Lévesque; de L'embrumé de Viard et Zacharias, par Vianney Gallant; de Neige noire de Hubert Aquin, par Jacquelin Marcheterre. Il fait plaisir de souligner que ces quatre études, chacune ayant des références, une écriture et des points de vue qui sont les siens, furent d'abord des travaux fournis à l'occasion d'un séminaire de deuxième cycle intitulé Études de littérature comparée, séminaire que j'ai dirigé à quelques reprises. Hamlet et Palimpsestes furent là régulièrement à l'honneur - que les étudiantes et étudiants de ce séminaire veuillent bien lire dans ce numéro un témoignage à leur égard.

Renald Bérubé 


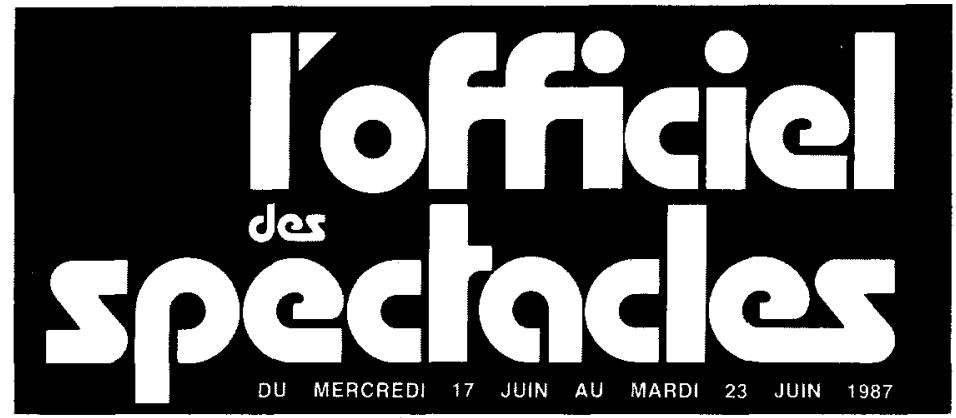

\section{cette semaine № $21122 \mathrm{~F}$}
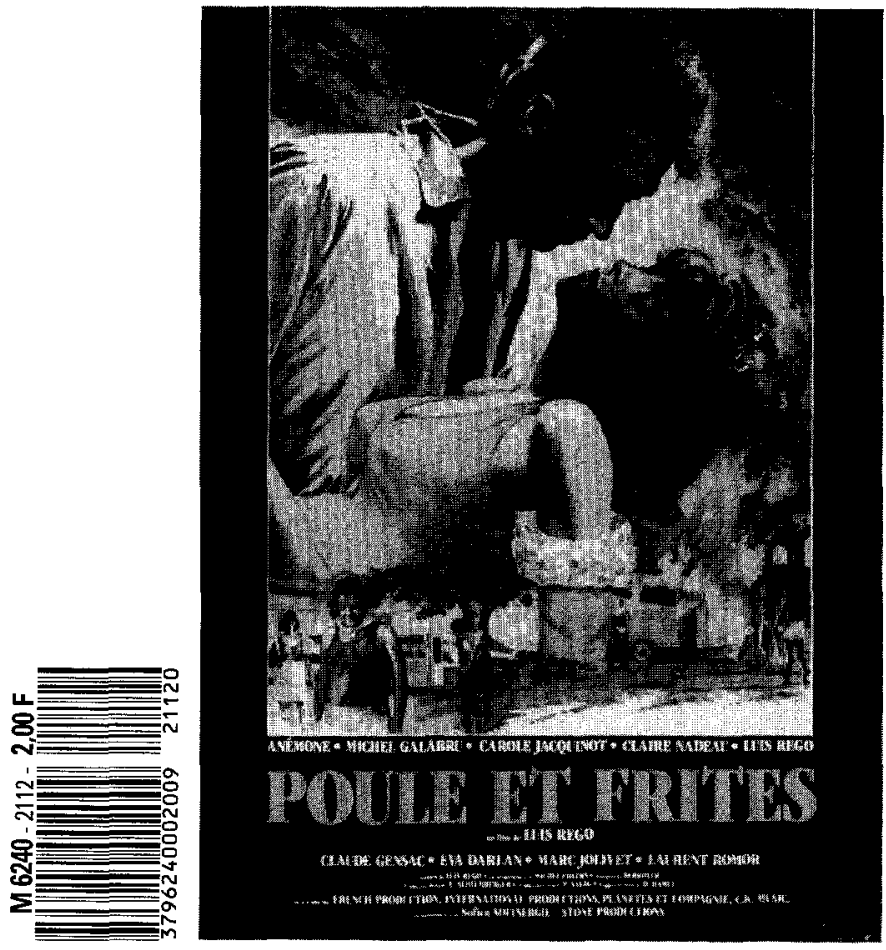

PALIMPSESTE: Au tempe qu'emporte le vent Au vent qu'emporte le tempg ou

Le poulet frit à $1 \mathrm{a}, \ldots$ ? 